\title{
Intraductal proliferative lesions of the breast: morphology, associated risk and molecular biology
}

\author{
Ian O Ellis
}

Department of Histopathology, University of Nottingham, Molecular Medical Sciences, Nottingham City Hospital, Nottingham, UK

\begin{abstract}
The main objectives of this review are (1) to review the histological criteria and terminology for usual ductal hyperplasia, atypical ductal hyperplasia, flat epithelial atypia and ductal carcinoma in situ; (2) to provide an overview of the risk of breast cancer associated with these various lesions; (3) to discuss established and emerging data on the molecular biology and pathogenesis of these lesions and (4) to discuss management when detected in diagnostic needle core and surgical diagnostic specimens.

Modern Pathology (2010) 23, S1-S7; doi:10.1038/modpathol.2010.56
\end{abstract}

Keywords: breast; ductal carcinoma in situ; atypical hyperplasia; columnar cell lesions; usual hyperplasia

\section{The histological criteria and terminology for usual ductal hyperplasia, atypical ductal hyperplasia, flat epithelial atypia and ductal carcinoma in situ}

Introduction and Background to Terminology

Intraductal proliferative lesions of the breast have traditionally been divided into three categories: usual ductal hyperplasia (UDH), atypical ductal hyperplasia (ADH) and ductal carcinoma in situ (DCIS). ${ }^{1}$ It should be noted, however, that the term 'DCIS' encompasses a highly heterogeneous group of lesions that differ with regard to their mode of presentation, histopathological features, biological markers and risk for progression to invasive cancer. In most cases, the histopathological distinction between different types of intraductal proliferation can be made on morphological grounds alone, particularly with standardization of histopathological criteria. ${ }^{2}$ However, even then, the distinction between some of the lesions (particularly between $\mathrm{ADH}$ and some low-grade forms of DCIS) remains problematic. In addition, population-based mammography screening has resulted in increased detection of lesions that show cytological atypia with or

Correspondence: Dr IO Ellis, MD, Department of Histopathology, Nottingham City Hospital, Hucknall Road, Nottingham NG5 1PB, UK.

E-mail: ian.ellis@nottingham.ac.uk

Received 12 February 2010; accepted 12 February 2010 without intraluminal proliferation but do not fulfill the diagnostic criteria for any of the existing categories. Those lesions lacking intraluminal projection have been described in the past as clinging carcinoma and more recently referred to under a variety of names, including atypical cystic lobules, atypical columnar alteration with prominent apical snouts and secretions; however, the terms columnar cell change for non-atypical lesions and flat epithelial atypia (FEA) for those with atypia have become the preferred terms for such lesions. ${ }^{3}$

The existing system for the classification of intraductal epithelial proliferations assumes a spectrum from usual epithelial hyperplasia through ADH and low-grade DCIS to high-grade disease. Recently published work using comparative genomic hybridization to investigate DCIS of the breast has prompted the proposal of a hypothetical model for the pathogenesis of DCIS, which recognizes genetic lesions associated with particular morphological subtypes. ${ }^{4-6}$ These data also indicate that ADH/ low-grade DCIS is more closely related to lobular in situ neoplasia than to high-grade DCIS. Thus, new molecular genetic techniques are showing that current dogma is untrue: (1) the fundamental separation of lobular carcinoma in situ (LCIS) from DCIS may not be appropriate; (2) distinguishing ADH from low-grade DCIS is illogical and (3) the assumption that the DCIS is a spectrum of the same disease is flawed. It is likely that some of these new methods will form the basis of a revised system 
of classification with an underlying molecular genetic basis while maintaining clinical relevance.

\section{Hyperplasia vs Neoplasia}

As with diseases elsewhere in the body, distinguishing hyperplasia from neoplasia in the breast is based on identification of a clonal cell process. Clonality is recognized by uniformity of morphology and phenotype and markers such as cytokeratin expression or hormone receptor expression can be used. Although usual epithelial hyperplasia is morphologically and phenotypically heterogeneous, ADH and established DCIS have a homogeneity of cell type and marker expression. In addition, studies of loss of heterozygosity (LOH) in low-grade DCIS and ADH have shown similar genetic lesions that is interpreted as confirmatory evidence that these are clonal processes and both therefore fulfill the basic concept of neoplasia. The frequency of $\mathrm{LOH}$ in cases of usual hyperplasia is much lower and typically at randon sites. ${ }^{7}$

The conceptual distinction between benign neoplasia and in situ malignancy in the intraductal epithelial proliferations of the breast has been arbitrarily drawn at the boundary between $\mathrm{ADH}$ and low-grade DCIS. This may not be the appropriate place. Indeed morphological, immunohistochemical and genetic studies indicate that it is more appropriate to draw the boundary between usual epithelial hyperplasia and $\mathrm{ADH}^{8}$

\section{Atypical Ductal Hyperplasia}

Distinction between DCIS and ADH is based on evidence derived from many series including studies by Page et $a l^{9}$ These have been supported by other studies, such as the Nurses' Health Study. ${ }^{10}$ It is clear that $\mathrm{ADH}$ is a rare condition being seen in $4 \%$ of symptomatic benign biopsies, although it is more common in association with screen-detected benign microcalcifications and is seen most commonly as an incidental finding.

The diagnostic criteria used to define $\mathrm{ADH}$ are imperfect. ADH was described initially based on exclusion rather than positive criteria, that is the recognition of some but not all of the features of DCIS (as well as the lack of the characteristics of usual type epithelial hyperplasia). This definition of ADH has been updated and, although the diagnosis stills rests on an absence of all the features of DCIS, additional supporting features have been described. Page's view that the cellular changes of DCIS are present but occupy less than two separate duct spaces is widely accepted. Others use a $2 \mathrm{~mm}$ cutoff; a lesion less than $2 \mathrm{~mm}$ in maximum dimension being classified as $\mathrm{ADH}$ and a larger area as DCIS. These criteria recognize essentially the same lesions. In essence, ADH is usually small and focal, measuring less than
2-3 mm. Larger foci are accepted if associated with a radial scar/complex sclerosing lesion or a papilloma.

There are three components to the diagnosis of $\mathrm{ADH}$, namely the architectural pattern, cytology and disease extent. $\mathrm{ADH}$ is formed from a uniform population of small- or medium-sized round, cuboidal or polygonal hyperchromatic cells, which are regularly arranged. The nuclei are evenly distributed and may form a rosette-like pattern. Single small nucleoli only are present. Mitoses, particularly abnormal forms, are infrequently seen. Geometric spaces are noted and, in the cribriform type, the cells are arranged at right angles to the bridges formed. Micropapillary ADH is also recognized and a solid pattern may very rarely be seen. Small foci of necrosis may rarely be identified in $\mathrm{ADH}$ and do not indicate that the process should be classified as DCIS.

At present it is recommended that the diagnosis of ADH should be restricted to lesions that show the features described by Page et al to which the quantified risk of developing breast carcinoma is linked. ${ }^{11,12}$ Even then the diagnosis of $\mathrm{ADH}$ should be made with caution and only if low-grade DCIS has been seriously considered in the differential diagnosis. Lesser changes for which the possible classification lies between florid UEH and $\mathrm{ADH}$ are less relevant with regard to a risk of developing breast carcinoma and should not be classified as ADH. However it should also always be borne in mind that a proliferation at the edge of a biopsy may represent the periphery of a more established lesion of DCIS and further excision of the adjacent tissue may be warranted.

The major problem of ADH is the difficulty in achieving acceptable levels of concordance or consistency in diagnosis. Various strategies have been used to try to improve its recognition, including revision of the criteria, providing a more positive basis for recognition and education and emphasis of the use of one system by all for diagnosis. ${ }^{13}$ Despite the adoption of such principles, the reliable classification around the boundaries of $\mathrm{ADH}$ and the 'borderline' epithelial intraductal proliferations in the breast remains elusive in several, although not all, studies.

\section{Defining Columnar Cell Change and Flat Epithelial Atypia}

Columnar cell change (without atypia)

The classic columnar cell lesion (CCL) is composed of acini internally lined by cells that are tall and snouted in a manner similar to that observed in tubular carcinoma (TC). ${ }^{3,14}$ Commonly this is associated with luminal secretions and/or microcalcifications. If there is a piling up of several layers the term 'columnar hyperplasia' is used, assuming that the stratification is real, as opposed to artifactual as 
a consequence of cross-cutting. There is morphological diversity within these groups, for example, the hyperchromasia of the nuclei can vary, as well as nuclear shape and the 'tallness' of the cells; thus, in some cases some lesions could be argued as more cuboidal than columnar.

\section{Flat epithelial atypia}

In columnar cell change with atypia and columnar cell hyperplasia with atypia, cytological abnormalities are present, and these lesions are presently group together under the term FEA. There is therefore morphological diversity in this group of lesion; in some cases the atypia manifests as relatively uniform rounded, evenly spaced nuclei with a similar cytomorphology to that displayed by the cells of low and some intermediate grade DCIS. In others, distinct nuclear pleomorphism is evident, but falling short of high nuclear grade, but, equally, without the relative nuclear uniformity seen in CCC. Some FEA lesion display a distinctly endometrioid appearance with tightly packed tall hyperchromatic fusiform nuclei.

\section{Markers in columnar cell lesions}

Unlike the normal breast and UDH, where ER and PR staining is heterogeneous and limited to about $10-15 \%$ of cells, in columnar cell change and related lesions there is extensive, and uniform, positivity of the nuclei of all lesional cells. Most cells stain positively for low-molecular-weight cytokeratins such as keratin 19. Unlike UDH, the lesional cells do not express basal markers such as CK 5/6. There is limited diagnostic value in undertaking immunohistochemical assessment with any known markers, beyond what thorough morphology examination with routine hematoxylin and eosin staining can provide.

\section{Ductal Carcinoma In Situ}

DCIS is defined as a proliferation of malignant epithelial cells within the breast parenchymal structures with no evidence of invasion across the basement membrane. This lack of invasive foci may be confirmed with immunohistochemical assessment for the presence of myoepithelial cell (eg, smooth muscle actin, smooth muscle myosin) or basement membrane (type IV collagen, laminin). Pure DCIS accounts for $15-20 \%$ of breast cancers compared with only $5 \%$ of cases before the advent of breast cancer screening. ${ }^{2}$

DCIS is usually a unicentric disease process, as shown by elegant three-dimensional studies showing that only one region of the breast is involved in the vast majority of cases and two thirds of tumors involve only one quadrant. ${ }^{15}$ However, the natural history of DCIS is not well understood as it has largely been extrapolated from historical series and reassessment of lesions previously misdiagnosed, most of which were low grade. The numbers in these series are low; in Page's series 28 patients were found to have DCIS from the 11760 biopsies reviewed and none were of comedo type. ${ }^{16}$ Studies suggest that up to $50 \%$ of patients with microscopic foci of DCIS develop invasive carcinoma. The invasive lesion occurs in the same area as the original lesion indicating a precursor process. Series of cases of DCIS not completely excised have also been reviewed and these indicate that progression to invasion is related to the subtype of DCIS; comedo disease progresses into invasive carcinoma both more often and more rapidly than low-grade DCIS. ${ }^{2,17}$

Several systems for subdividing DCIS have been described. The traditional classification based on a combination of architectural growth pattern and cytological features, provides poor reproducibility, with up to $30 \%$ of cases in multicenter trials requiring reclassification. The current international consensus system classifies DCIS as high, low or intermediate grade based on cytonuclear features. This system has clinical relevance and shows reasonable reproducibility. Several other systems for classification of DCIS have been proposed, including categorization based on nuclear grade and necrosis. Silverstein et $a 1^{18}$ have grouped DCIS into high grade, non-high grade with necrosis and non-high grade without necrosis and found an association between the subtypes and local recurrence and disease-free survival.

\section{Recommended system for classification of DCIS}

\section{High Nuclear Grade DCIS}

This is the easiest pattern to recognize. ${ }^{4}$ Cells have pleomorphic, irregularly spaced and usually large nuclei exhibiting marked variation in size, irregular nuclear contours, coarse chromatin and prominent nucleoli. Mitoses are frequent and abnormal forms may be seen. High-grade DCIS may exhibit several growth patterns; most commonly as a solid sheet of cell lining the duct and with comedo-type central necrosis that frequently contains deposits of amorphous calcification.

\section{Intermediate Nuclear Grade DCIS}

This type cannot be assigned easily to the high or low nuclear grade categories. The nuclei show mild to moderate pleomorphism, which is less than that seen in the large cell variety, but lacks the monotony of the small cell type. The growth pattern may be solid, cribriform or micropapillary.

\section{Low Nuclear Grade DCIS}

These types are composed of monomorphic, evenly spaced cells with roughly spherical, centrally placed nuclei and inconspicuous nucleoli. The 
nuclei are usually, but not invariably, small. Mitoses are few and there is rarely individual cell necrosis. The cells are generally arranged in micropapillary and cribriform patterns.

\section{Mixed Types of DCIS}

A proportion of cases of DCIS exhibit features of more than one histological subtype. One of the advantages of classifying DCIS according to nuclear grade is that when there are variations in growth pattern but a dominant cell type, the lesion can be classified on the basis of nuclear grade. Foci of differing nuclear grade may be seen, but such variation in cell type is unusual, although if present, the case should be classified according to the highest nuclear grade.

\section{Risk of progression to invasive breast cancer and related issues}

The existing classification systems used to identify histological risk lesions for development of breast cancer are based on conventional morphological criteria. They include a range of epithelial proliferative lesions extending from the common epithelial hyperplastic process, ductal hyperplasia of usual type (UEH), to high-grade DCIS. Intermediary forms include intermediate and low-grade forms of DCIS and ADH. The lobular neoplastic epithelial proliferations, atypical lobular hyperplasia (ALH) and LCIS form a similar parallel spectrum of risk lesions. Clinical follow-up studies have indicated that these intraductal proliferative lesions are associated with different levels of risk for subsequent development of invasive breast cancer, that ranges from approximately 1.5 times that of the reference population for UDH, 4- to 5-fold (range, 2.4- to 13.0-fold) for $\mathrm{ADH}$, and 8- to 10-fold for DCIS. ${ }^{9,10}$ The main clinical significance of the diagnosis of $\mathrm{ADH}$ lies in the increased risk of invasive breast carcinoma that is about $4-5$ times that of the general population and may be even greater for premenopausal women This risk has been shown in some but not all studies to be further increased if the patient has a first-degree relative with breast cancer (10 times increased risk). ${ }^{9}$

CCLs of the breast encompass a morphological spectrum within a wider range of lesions that may include carcinoma. However, the overall biological significance of these lesions is unknown. There is supporting evidence for a precursor role, including an association with TC, the finding that lesions with these characteristics are found more commonly in cancerous rather than noncancerous breasts, are often continuous with cancerous lesions in breast cancer biopsies commonly coexist with in situ lobular neoplasia (LN) and share similar cytological characteristics and immunohistochemical profile with coexistent malignancies. Despite the above, the certainty and speed of progression of these lesions to malignancy is unknown. Eusebi et $a l^{19}$ described 25 cases of what was called low-grade clinging carcinoma of flat type, but which appears morphologically equivalent to columnar change with atypia/FEA. During an average follow-up interval of 19 years, none of the 25 women with low-grade clinging carcinoma of flat type developed invasive carcinoma and only one had a recurrence, which was described as consisting of low-grade clinging carcinoma of flat type. ${ }^{19}$

At a more fundamental level there is evidence that, in general, there is a progressive accumulation of allelic damage in columnar cell change with atypia though to DCIS and invasive carcinoma, implying a molecular continuum from at least some lesions with a columnar morphology (which is not the case for UDH and carcinoma). ${ }^{5}$

Taken together, these data imply that precursor columnar lesions can often coexist with established malignancy, but that the biological progression of an individual columnar lesion may be slow. As a consequence, the finding of columnar change with atypia/FEA in a core may predict for presence of malignancy in the breast. In contrast this does not imply that columnar change with atypia/FEA extending to an excision margin in a surgical specimen is a significant risk for cancer recurrence and it is not recommended at present that further surgery is required in this situation.

\section{Molecular pathology of intraductal lesions}

Recent molecular profiling studies have shown the existence at least five different BC subtypes, each with different clinical outcomes. ${ }^{20,21}$ These include luminal (A and B), basal, HER2 and normal-like subtypes. The luminal subtype 'A' have higher levels of estrogen receptor $\alpha$ and a better survival outcome compared with luminal subtypes 'B'. The basal and HER2 subtypes are generally of higher grade than the luminal subtypes. Furthermore, there is convincing genetic evidence to suggest that lowgrade (LGBC) and high-grade DCIS and invasive breast cancers evolve through distinct evolutionary pathways. ${ }^{4,8,22}$ LGBCs are usually diploid/neardiploid and harbor recurrent loss of chromosome $16 \mathrm{q}$ and gains of chromosome 1q. In contrast, highgrade breast cancers are usually aneuploid with complex genetic profiles and infrequent deletion of 16q. In high-grade breast cancers, even when loss of $16 \mathrm{q}$ is present, the underlying genetic mechanism appears to be distinct from that seen in LGBCs. Taken together, these findings suggest that progression from LGBC to high-grade breast cancers is an unlikely biological phenomenon and that LGBCs are a distinct class of breast cancer. How do more traditional histological system relate to these emerging classes? Which histological types of breast 


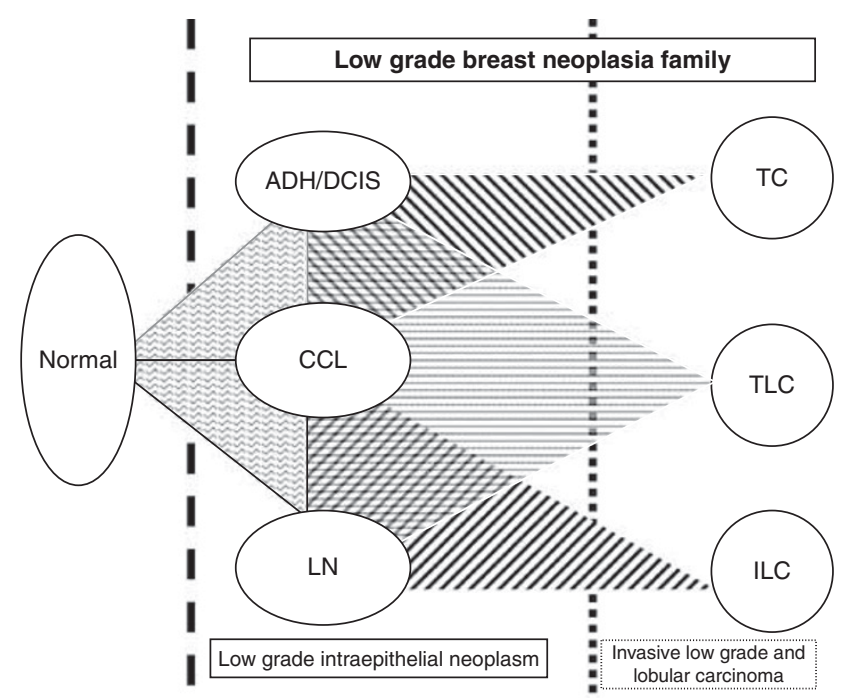

Figure 1 A schematic diagram to illustrate the evolutionary pathways of low grade breast in situ and invasive neoplasia.

cancer are found in or form the luminal subtype of breast cancer?

We have recently proposed the concept of a family of related low nuclear grade precursor, in situ and invasive neoplastic lesions of the breast, based on the significant coexistence of CCLs, FEA, LN and $\mathrm{ADH} /$ low-grade DCIS with invasive TC, tubulolobular carcinoma (TLC) and classic invasive lobular carcinoma (ILC) (Figure 1). ${ }^{6}$ This view is further supported by the observation that FEA, ADH/lowgrade DCIS, LN and invasive LGBCs have similar molecular genetic and immunophenotype characteristics which are distinct from those seen in highgrade breast cancers. It has therefore been proposed that FEA is a common nonobligate precursor of lowgrade invasive ductal cancer and ILC and that taken together, these lesions may represent a family of precursor, in situ and invasive neoplastic lesions belonging to the luminal ' $\mathrm{A}$ ' subclass of $\mathrm{BC}$. If this is correct then how can the strikingly different morphology of LCIS compared with low-grade DCIS and invasive TC when compared with classical ILC be explained? We speculate that alteration of E-cadherin $(C D H 1)$, the tumor suppressor gene localized on chromosome 16q21 and which is frequently seen in ILC (in contrast to CCLs, ADH/low-grade DCIS, TC, ICC, low-grade IDC, and TLC, which have normal E Cadherin expression), is a secondary event in tumorigenesis which is associated with the development of lobular morphology/differentiation.

\section{Future Classification}

Distinction between these entities is based on evidence derived from a number of studies and their international acceptance reflects the relative consistency of data emerging from the various studies with respect to risk of subsequent development of invasive breast cancer. Problems have arisen with difficulty in achieving acceptable levels of concordance or consistency in diagnosis between pathologists for lesions such as ADH which is recognized largely on criteria of exclusion rather than positive criteria; that is, recognition of some but not all features of DCIS and lack of the characteristics of usual type epithelial hyperplasia.

Ideally future classification systems for epithelial proliferative diseases of the breast will be reproducible between centers, and take account of clinical, morphological, phenotypic and genetic evidence. Clinical management will be based on the relevance and extent of these specific clinicopathological entities. Hallmark genetic lesions will have been identified for many of the common and indeed uncommon lesions we currently recognize. These abnormalities will underpin the basis of the classification systems and disease subclassification will be based on acquisition of additional molecular genetic changes and disease extent.

\section{Management when detected in diagnostic needle core and surgical diagnostic speci- mens}

It is important to remember that histological examination of core biopsy samples is performed to fulfill the assessment process role by giving a pathology category classification (B1-5) for clinical management purposes based on the triple approach to breast disease diagnosis and not designed to give a definitive diagnosis, although this is possible in the majority of cases. ${ }^{23}$ Thus although most core biopsy samples can be readily categorized as normal, benign or malignant, it must be recognized that a small proportion (probably less than 10\%) of samples cannot. In Europe a set of reporting guidelines have been devised in recognition of this and can be used for all screen-detected and symptomatic lesions (microcalcification, architectural deformities and mass lesions). ${ }^{23}$ It is also important to remember that although there are five reporting categories similar to those used in fine needle aspiration cytology, these are not equivalent.

These categories are designed to take account purely of the histological nature of the specimen and not the clinical or imaging characteristics. Similarly it is not feasible for pathology interpretation to judge independently whether a sample is adequate and from the mammographic lesion. This judgment requires multidisciplinary discussion. For these reasons there is no inadequate biopsy category for core biopsy specimens. In certain situations, particularly epithelial proliferation and papillary lesions, the choice of diagnostic category should be based on the features present in the sample.

\section{Needle Core Biopsy of Breast: Diagnostic Categories}

B1-Normal (breast) tissue/inadequate sample B2-Benign lesion 
B3-Lesion of uncertain malignant potential (includes atypical epithelial proliferations, papillary lesions, sclerosing lesion, phyllodes tumor)

B4-Suspicious of malignancy

B5-Malignant

(a) In situ

(b) Invasive

(c) Unclassified

B1 normal tissue indicates that no obvious breast lesion is present; appropriate for any type normal tissue with comment on presence of breast parenchymal structures and correlative features such as microcalcification; may be consistent with some lesions such as lipoma or hamartoma.

B2 benign breast lesion, part of a recognizable benign lesion is present in the specimen such as fibroadenoma; sclerosing adenosis; fibrocystic change and cysts; some papillary lesions, such as inflammatory condition.

B3 benign of uncertain malignant potential, part of a recognizable benign breast lesion known to be associated with malignancy is present in the biopsy: sclerosing lesions (Radial scar, CSL), some papillary lesions, atypical epithelial proliferations including $\mathrm{ADH}$ and LN (ALH and LCIS) and phyllodes tumor.

B4 suspicious of malignancy, used when only microfocal changes resembling malignancy are present such as a single duct space with high-grade changes (DCIS), or if artifact changes such as crushing preclude definative assessment.

B5 malignant, unequivocal features of malignancy present; can distinguish between in situ and invasive disease.

\section{Atypical Epithelial Proliferations (ADH)}

Included under a B3 classification are atypical epithelial hyperplastic lesions where a uniform population of cells arranged in an appropriate manner involves one duct space or partially involves two or more duct spaces. ${ }^{23}$ These appearances should be sufficiently structured to raise the possibility of low-grade DCIS but insufficient in the tissue available to fulfill the diagnostic criteria. There is a range of severity from those that are insufficient for a definite diagnosis of DCIS but highly suspicious to those that show only a minor degree of atypia, which is normal of an architectural nature, and requires further assessment and judgment of appropriate categorization as B3 or B4.

The definition of ADH is derived from surgical resection specimens and relies on a combination of histological, morphological and size extent criteria. For this reason, accurate diagnosis of $\mathrm{ADH}$ is not possible on core biopsy. ${ }^{23}$ It has however been shown that core biopsy samples that include atypical intraductal epithelial proliferative foci, of insufficient extent for classification as DCIS, on subsequent surgical resection may form part of an established in situ neoplastic lesion with or without associated invasion. This view is based on several studies that describe the subsequent surgical diagnoses in cases described as $\mathrm{ADH}$ in preoperative core biopsy. In over $50 \%$ of cores surgical excision biopsy has shown either in situ or invasive carcinoma. This is not surprising as ADH is basically defined as an intraductal epithelial proliferation showing the features of low-grade DCIS, but in less than two duct spaces or less than $2 \mathrm{~mm}$ in diameter. The limited tissue sampling that can be undertaken by core biopsy guns (often by stereotactic methods for foci of microcalcification) may thus provide insufficient material for definitive diagnosis of lowgrade DCIS if only a few ducts spaces are obtained. In these cases a diagnosis of atypical intraductal epithelial proliferation and a classification of B3 of uncertain malignant potential or B4 suspicious of malignancy should be made dependant on the severity and extent of the lesion.

\section{Ductal Carcinoma In Situ}

The diagnosis of DCIS on core biopsy does not exclude invasive carcinoma and that although the positive predictive value of stereotaxic core biopsy for the presence of invasion is reported to be $98 \%$, the negative predictive value is only about $80 \% .{ }^{23}$

\section{Disclosure/conflict of interest}

The author declares no conflict of interest.

\section{References}

1 Page DL, Dupont WD. Anatomic indicators (histologic and cytologic) of increased breast cancer risk. Breast Cancer Res Treat 1993;28:157-166.

2 Van Dongen JA, Holland R, Peterse JL, et al. Ductal carcinoma in-situ of the breast; second EORTC consensus meeting. Eur J Cancer 1992;28:626-629.

3 Schnitt SJ, Vincent-Salomon A. Columnar cell lesions of the breast. Adv Anat Pathol 2003;10:113-124.

4 Simpson PT, Reis-Filho JS, Gale T, et al. Molecular evolution of breast cancer. J Pathol 2005;205: 248-254.

5 Simpson PT, Gale T, Reis-Filho JS, et al. Columnar cell lesions of the breast: the missing link in breast cancer progression? A morphological and molecular analysis. Am J Surg Pathol 2005;29:734-746.

6 Abdel-Fatah TMA, Powe DG, Hodi Z, et al. High frequency of coexistence of columnar cell lesions, lobular neoplasia and low grade ductal carcinoma in situ with invasive tubular carcinoma and invasive lobular carcinoma. Am J Surg Pathol 2007;13:417-426.

7 Lakhani SR, Collins N, Stratton MR, et al. Atypical ductal hyperplasia of the breast: clonal proliferation with loss of heterozygosity on chromosomes 16q and 17p. J Clin Pathol 1995;48:611-615.

8 Ellis IO, Pinder SE, Elston CW. A critical appraisal of existing classification systems of epithelial hyperplasia and in situ neoplasia of the breast with proposals 
for future methods of categorization: where are we going? Semin Diagn Pathol 1999;16:202-208.

9 Page DL, Dupont WD, Rogers LW, et al. Atypical hyperplastic lesions of the female breast. A long-term follow-up study. Cancer 1985;55:2698-2708.

10 Marshall LM, Hunter DJ, Connolly JL, et al. Risk of breast cancer associated with atypical hyperplasia of lobular and ductal types. Cancer Epidemiol Biomarkers Prev 1997;6:297-301.

11 Pathology Reporting of Breast Disease. NHSBSP and RCPath. A Joint Document Incorporating the Third Edition of the NHS Breast Screening Programme's Guidelines for Pathology Reporting in Breast Cancer Screening and the Second Edition of The Royal College of Pathologists' Minimum Dataset for Breast Cancer Histopathology. NHSBSP Publication No. 58, 2005.

12 European, Community. European Guidelines for Quality Assurance in Mammographic Screening, 2nd edn. Office for Official Publications of the European Communities: Luxembourg, 1996.

13 Schnitt SJ, Connolly JL, Tavassoli FA, et al. Interobserver reproducibility in the diagnosis of ductal proliferative breast lesions using standardized criteria. Am J Surg Pathol 1992;16:1133-1143.

14 Schnitt SJ. The diagnosis and management of preinvasive breast disease: flat epithelial atypia-classification, pathologic features and clinical significance. Breast Cancer Res 2003;5:263-268.

15 Holland R, Hendriks JHCL, Vebeek ALM, et al. Extent, distribution, and mammographic/histological correlations of breast ductal carcinoma in situ. Lancet 1990;335:519-522.
16 Page DL, Dupont WD, Rogers LW, et al. Continued local recurrence of carcinoma 15-25 years after a diagnosis of low grade ductal carcinoma in situ of the breast treated only by biopsy. Cancer 1995;76: 1197-1200.

17 Lagios MD. Duct carcinoma in situ. Pathology and treatment. Surg Clin North Am 1990;70:853-871.

18 Silverstein MJ, Lagios MD, Craig $\mathrm{PH}$, et al. A prognostic index for ductal carcinoma in situ of the breast. Cancer 1996;77:2267-2274.

19 Eusebi V, Feudale E, Foschini MP, et al. Long-term follow-up of in situ carcinoma of the breast. Semin Diagn Pathol 1994;11:223-235.

20 Sorlie T, Perou CM, Tibshirani R, et al. Gene expression patterns of breast carcinomas distinguish tumour subclasses with clinical implications. Proc Natl Acad Sci USA 2001;98:10869-10874.

21 Sorlie T, Tibshirani R, Parker J, et al. Repeated observation of breast tumor subtypes in independent gene expression data sets. Proc Natl Acad Sci USA 2003;100:8418-8423.

22 Buerger H, Mommers EC, Littmann R, et al. Ductal invasive G2 and G3 carcinomas of the breast are the end stages of at least two different lines of genetic evolution. J Pathol 2001;194:165-170.

23 Ellis IO, Humphreys S, Michell M, , et al., UK National Coordinating Committee for Breast Screening Pathology; European Commission Working Group on Breast Screening Pathology. Best Practice No 179. Guidelines for breast needle core biopsy handling and reporting in breast screening assessment. J Clin Pathol 2004; 57:897-902. 